\title{
Construction of a 3D mammary duct based on spatial localization of the extracellular matrix
}

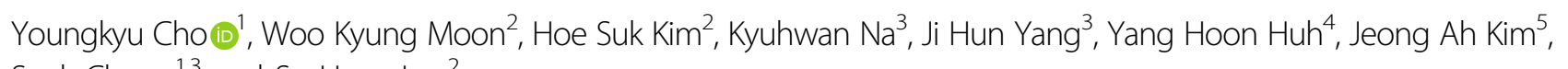
Seok Chung ${ }^{1,3}$ and Su Hyun Lee ${ }^{2}$

\begin{abstract}
Extracellular matrix (ECM)-based hydrogels can serve as scaffolds in reconstruction of physiologically relevant threedimensional (3D) in vitro models. Biocompatible or integrated hydrogels can be used to modulate ECM properties such as stiffness and composition for studies of cell-ECM interactions and morphogenesis. To this end, we developed a new type of spatially modified collagen type 1 hydrogel by convective addition of collagen type 1 solution. The matrix displayed properties that were distinct from those of a collagen type 1 hydrogel and recapitulated the morphology and function of mammary epithelium in a 3D microfluidic platform. In this ECM, mammary epithelial cells secreted laminin and exhibited self-assembly into a basement membrane. Thus, this spatially modified ECM offers biophysical features that can facilitate the construction of mammary epithelium and, by extension, that of various other epithelial types. Additionally, our reconstructed mammary duct can be used as an in vitro model for the study of early-stage breast cancer.
\end{abstract}

\section{Introduction}

The mammary duct branches from the mammary gland to transport milk for feeding infants contains a hierarchical epithelial structure and is surrounded by microenvironmental components such as an extracellular matrix (ECM), stromal cells (mainly fibroblasts and adipocytes), and blood and lymph capillaries ${ }^{1-3}$. Breast ECM is typically composed of collagen type I (COL1, the most abundant ECM component), laminin, collagen type IV (COL4), fibronectin, and other proteins ${ }^{4,5}$, and its properties of composition, density, and stiffness influence cell function and phenotype ${ }^{6-9}$ via regulation of gene expression, morphology, and cell migration and differentiation $^{10-12}$. For example, ECM density has been shown

Correspondence: Seok Chung (sidchung@korea.ac.kr) or

Su Hyun Lee (suhyune82@gmail.com)

'Department of IT Convergence, Korea University, 145 Anam-ro, Seoungbukgu, Seoul 02841, South Korea

2Department of Radiology, Seoul National University Hospital, 101 Daehak-ro, Jongno-gu, Seoul 03080, South Korea

Full list of author information is available at the end of the article. to play a role in tissue regeneration and maintenance ${ }^{13-15}$, and its porosity regulates cell migration and positioning ${ }^{16}$. Additionally, interactions among the other microenvironmental components regulate mammary epithelial branching through paracrine signaling of growth factors secreted by adipocytes ${ }^{17}$. These interactions affect not only epithelial development but also disease progression, including tumorigenesis ${ }^{2,18}$.

Microenvironmental components are the main focus of recently developed three-dimensional (3D) models ${ }^{4}$. In typical 2D models that use microwells, Transwells, or Petri dishes, cells are cultured on ECM-coated plastics and exhibit changes in gene expression, morphology, and cell-ECM interactions in response to soluble factors in the medium ${ }^{19,20}$. However, these models do not accurately reflect the complexity of microenvironmental components, which can be successfully recapitulated by in vitro $3 \mathrm{D}$ models ${ }^{21-24}$. In one such model, epithelial cell aggregates were embedded in ECM composed of COL1, Matrigel 
(MAT), or their mixture and formed spheroids with a hypoxic core and hollow microstructures known as acini $^{23,24}$ that developed apical-basal polarity when embedded in hydrogel and secreted milk protein, although no fluid flowed through the hollow structure of the mammary duct. Another model with a branched lumen was developed to mimic the hierarchy of mammary ducts on a basement membrane (BM) protein (MAT)-coated polydimethylsiloxane (PDMS) microfluidic channel surface ${ }^{21}$. Although the model simulated branched lumen structures on a BM, it had no other microenvironmental components since the epithelial cells were in direct contact with the PDMS surface. Another in vitro mammary duct was formed on a hydrogel with cocultured fibroblasts that included a BM and stromal cells but only on a notably stiff, highdensity $(6 \mathrm{mg} / \mathrm{ml})$ collagen surface located far from the in vivo mammary duct ${ }^{25}$.

Recently, in vitro microfluidic models have been developed that incorporate hydrogel with various ECM components such as COL1 (at a low density, i.e., $2 \sim 3 \mathrm{mg} /$ $\mathrm{ml}$ ), MAT, and a COL1/hyaluronic acid mixture ${ }^{26-32}$. These models were used to investigate interactions between cells and microenvironmental components of ECM. ECM hydrogel modified by growth factors or other ECM components to mimic $\mathrm{BM}^{33,34}$ were shown to be essential for the formation of stable endothelial or epithelial barriers in in vitro models. Recombinant BM (rBM) was generated by spatial modification of COL1 nanofibers within diluted MAT $^{34}$ and formed a stable endothelial monolayer with an enhanced endothelial barrier function resulting from "outside-in" signaling between laminin and integrin. The mammary duct is also surrounded by BM components on an interstitial COL1 matrix, and thus a similar in vitro strategy can be used to form a confluent mammary duct.

We previously reported a protocol for generating rBM, MAT-coated COL1 nanofibers ${ }^{34}$ because MAT is mostly composed of BM components such as laminin and COL4. In this study, we also attempted to control the physical properties of COL1 hydrogel with the homogeneous material COL1 instead of MAT to construct mammary epithelium based on reports that the physical properties of ECM can affect epithelial cell behavior ${ }^{13-15}$. Even without addition of BM components, we hypothesized that regulating the physical properties of COL1 hydrogel only with homogeneous material of COL1 (COL1-coated COL1 hydrogel) could aid in formation of a stable mammary duct. We present a new method for spatial modification of COL1 hydrogel by convective addition and localization of additional ECM components on precured COL1 hydrogel nanofibers, which changes the local physical/chemical properties of the COL1 hydrogel.

\section{Materials and methods}

\section{Fabrication of the microfluidic device}

A microfluidic device incorporating hydrogel was fabricated from PDMS (Sylgard 184; Dow Chemical, Midland, MI, USA) cured on an SU-8 patterned silicon wafer by conventional soft lithography. Cured PDMS was cut, and holes were constructed with a biopsy punch to form the device, which was autoclaved at $120^{\circ} \mathrm{C}$ for $20 \mathrm{~min}$ followed by overnight drying at $80^{\circ} \mathrm{C}$. The PDMS device and a cover glass were bonded together by oxygen plasma treatment (Femto Science, Yongin, Korea). The microchannels were coated with $2 \mathrm{mg} / \mathrm{ml}$ polydopamine solution for $2 \mathrm{~h}$ at $25^{\circ} \mathrm{C}$, washed with distilled deionized water and dried overnight at $80^{\circ} \mathrm{C}$ to render the channel surface hydrophobic. The dimensions of the microfluidic device are illustrated in Figure S1(a).

\section{Convective/diffusive ECM coating of the COL1 hydrogel}

Spatially modified ECM channels were filled with $2 \mathrm{mg} / \mathrm{ml}$ COL1 solution (Corning Inc., Corning, NY, USA) at pH 7.4 and incubated at $37^{\circ} \mathrm{C}$ for $30 \mathrm{~min}$. Preparation of the COL1 solution is described in our previous paper ${ }^{26}$. In brief, COL1 solution was diluted with $10 \times$ phosphate-buffered saline (PBS) (Lonza, Walkersville, MD, USA) and distilled deionized water. The mammary duct (central) and side channels were filled with $1 \times \mathrm{PBS}$ followed by storage at $37^{\circ}$ $\mathrm{C}$ in a $5 \% \mathrm{CO}_{2}$ incubator for $24 \mathrm{~h}$. After removal of the PBS in the channels by aspiration, the side and mammary duct channels were filled with $20 \mu \mathrm{l}$ of $1 \times \mathrm{PBS}$ and $60 \mu \mathrm{l}$ of 200 $\mu \mathrm{g} / \mathrm{ml}$ COL1, MAT (Corning Inc.), or laminin (Thermo Fisher Scientific, Waltham, MA, USA), which were diluted with 1× PBS. Convective flow from the center to side channels was generated by the height difference between the reservoirs (approximately $1.6 \mathrm{~mm}$, corresponding to a pressure difference of $14.6 \mathrm{~Pa}$ ). After incubation at $37^{\circ} \mathrm{C}$ and $5 \% \mathrm{CO}_{2}$ for $40 \mathrm{~min}$, the channels were sequentially washed twice with $1 \times$ PBS and filled with cell culture medium. For diffusive flow coating, the side and mammary duct channels were filled with $60 \mu \mathrm{l}$ of $1 \times \mathrm{PBS}$ and an equal volume of 200 $\mu \mathrm{g} / \mathrm{ml} \mathrm{COL1}$, respectively (Fig. 1d and Fig. S1(b)).

\section{Cell seeding in the microfluidic device}

HMT-3522 S1 nonneoplastic human mammary epithelial cells (HMECs) were prepared with the MEGM BulletKit (Lonza) in a culture dish (Corning Inc.). The mammary duct channel was filled with $60 \mu$ cell suspension $\left(2 \times 10^{6} \mathrm{cells} / \mathrm{ml}\right)$, and the device was incubated at $37^{\circ} \mathrm{C}$ and $5 \% \mathrm{CO}_{2}$ for $3 \mathrm{~h}$ to induce cell attachment to noncoated and COL1-, MAT-, or laminin-coated COL1 hydrogel in the channel. During the incubation, interstitial flow generated by the pressure difference between the mammary duct (filled with $60 \mu \mathrm{l}$ of cell suspension) and side channels (filled with $20 \mu \mathrm{l}$ of culture medium) induced attachment of HMECs on COL1 hydrogel, as 
(a)

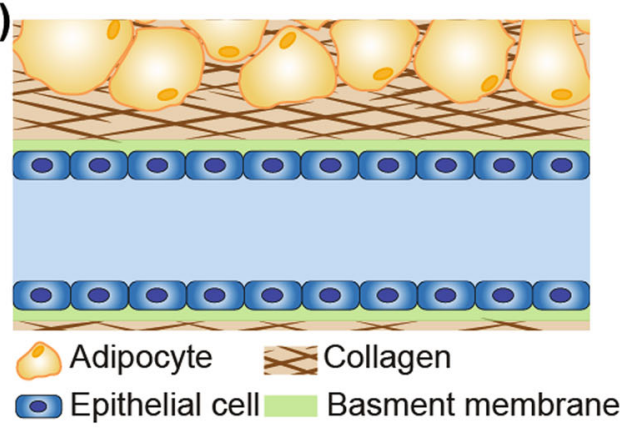

(b)

(c)
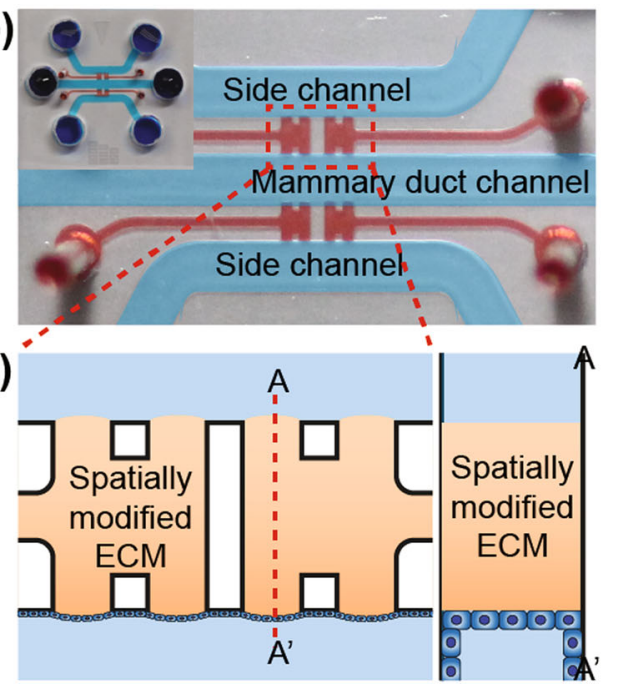

(d)
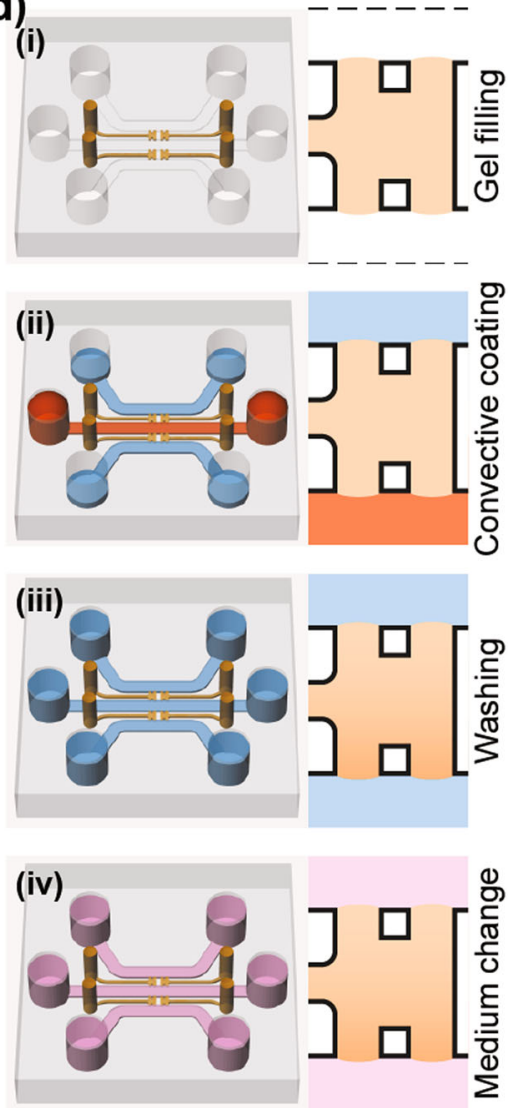

Fig. 1 Microfluidic device for spatial modification of the ECM and construction of 3D mammary duct. a Schematic illustration of a mammary duct composed of adipocytes, epithelial cells, interstitial matrix, and BM. b Photograph of the microfluidic device for 3D in vitro mammary duct remodeling. c Top and cross-sectional views of the microfluidic device focused on spatially modified ECM and the mammary duct channel. d Schematic illustration of the protocol for convective coating of the COL1 hydrogel incorporated in the microfluidic device. (i) COL1 hydrogel incorporation in microfluidic device, (ii) Convective coating on COL1 hydrogel, (iii) Washing convective coating solutions, (iv) filling cell culture medium

reported in ref. ${ }^{34}$. Nonattached cells were washed away with cell culture medium. The medium in the channel was refreshed daily. HMECs were cultured in the microfluidic device for 6 days.

Fluorescence labeling of the COL1 hydrogel and preparation of a fluorophore-conjugated COL1 coating solution

COL1 incorporated into the device was labeled with $0.1 \mathrm{mg} / \mathrm{ml}$ Alexa Fluor $488 \mathrm{~N}$-hydroxysuccinimide (NHS) ester (Thermo Fisher Scientific) at $37^{\circ} \mathrm{C}$ and $5 \%$ $\mathrm{CO}_{2}$ for $1 \mathrm{~h}$ and washed three times with $1 \times$ PBS. To visualize the COL1 coating, tetramethylrhodamine (TRITC)-conjugated COL1 solution was prepared according to a standard protocol ${ }^{35}$.

\section{Computational simulation of the COL1 coating procedure}

The concentration profile of the COL1 coating solution in the precured COL1 hydrogel was simulated using
Multiphysics v.5.2 software (COMSOL, Stockholm, Sweden). The diffusion coefficients for the COL1 solution in water and in the precured COL1 hydrogel were assumed to be $7.3 \times 10^{-12}$ and $7.21 \times 10^{-12} \mathrm{~m}^{2} / \mathrm{s}$, respectively. In the convective coating, the Darcy permeability in the precured COL1 hydrogel was assumed to be $8.9 \times 10^{-15} \mathrm{~m}^{2}$ (Fig. S3 and Table S1). The simulation result presents the position of individual COL1 molecules in the coating solution up to $40 \mathrm{~min}$, without considering the curing procedure.

\section{Imaging by transmission electron microscopy (TEM)}

To prepare samples for TEM imaging, the microfluidic devices were bonded with the PDMS membrane using a weak oxygen plasma treatment (30 s) instead of using a cover glass to detach the device from the bottom surface. Samples were fixed overnight at $4{ }^{\circ} \mathrm{C}$ with $2.5 \%$ glutaraldehyde in 0.15 M HEPES buffer. For postfixation, samples were treated with $0.1 \mathrm{M}$ sodium cacodylate trihydrate- 
buffered $1 \% \mathrm{OsO}_{4}$ solution at room temperature for $1 \mathrm{~h}$ and contrasted by treatment with $0.5 \mathrm{M}$ Trizma maleatebuffered $0.5 \%$ uranyl acetate at room temperature for $1 \mathrm{~h}$ in the dark. The samples were dehydrated in a graded series of ethanol solutions (50, 70, 95, 100, and 100\%) for $15 \mathrm{~min}$. Samples were embedded in the device channels using an epoxy medium kit (Sigma-Aldrich, St. Louis, MO, USA). The device was detached from the PDMS membrane, and the embedded sample was separated from the device and cut into ultrathin sections on an ultramicrotome and mounted on 200-mesh grids. The sections were contrasted with uranyl acetate and lead citrate and observed with a Bio-HVEM system (JEM-1000 BEF and JEM 1400 Plus) and a JEM 1010 TE microscope (JEOL, Tokyo, Japan).

\section{Measurement of ECM properties}

COL1 nanofibers appear as dots in the TEM images. The nanofiber diameter was measured according to the short axis length of the dots, and the interfiber distance was calculated as the distance between dots. The fiber number was counted in randomly selected $30-\mu \mathrm{m}^{2}$ areas of the ECM. Measurements were collected using ImageJ software (National Institutes of Health, Bethesda, MD, USA).

\section{Immunofluorescence labeling and confocal imaging}

The devices cultured with HMECs were fixed with $4 \%$ paraformaldehyde at room temperature for $20 \mathrm{~min}$. Cells were permeabilized and solubilized by incubation with $0.1 \%$ Triton X-100 for $10 \mathrm{~min}$. The samples were blocked with 20\% Block Ace (Dainippon Seiyaku, Osaka, Japan) in PBS for $1 \mathrm{~h}$ followed by washing with $1 \times$ PBS. The microfluidic channels were filled with solutions of primary antibody, including rabbit polyclonal anti-zonal occludens (ZO)-1, anti-Claudin 1, anti-Claudin 5, and anti-Laminin and mouse polyclonal anti-integrin $\alpha 6$ (all from Abcam, Cambridge, UK) and rabbit polyclonal anti-E-cadherin (Santa Cruz Biotechnology, Santa Cruz, CA, USA) antibodies, and incubated for $2 \mathrm{~h}$. After washing, Alexa Fluor 488-conjugated goat anti-rabbit and Alexa Fluor 568conjugated goat anti-mouse secondary antibodies, rhodamine-phalloidin (Thermo Fisher Scientific), and 4',6-diamidino-2-phenylindole (Sigma-Aldrich) were introduced into the channels followed by incubation for 2 $\mathrm{h}$. Images were acquired on a fluorescence microscope (Axio Observer D1; Carl Zeiss, Oberkochen, Germany) and a confocal laser scanning microscope (LSM700; Carl Zeiss, Oberkochen, Germany).

\section{Quantitative real-time (qRT)-PCR analysis}

Total RNA was isolated from the microfluidic device with the RNeasy Mini kit (Qiagen, Valencia, CA, USA). The RNA concentration was measured and normalized on a Nanodrop spectrometer (Thermo Fisher Scientific). cDNA was synthesized from the RNA using a high- capacity RNA-to-cDNA kit (Applied Biosystems, Foster City, CA, USA), and qRT-PCR was performed using a QuantiTect SYBR Green PCR kit (Qiagen) on a StepOne Real-Time PCR system (Applied Biosystems) with the following primer sets: laminin unit chain (LAMA)1 (forward, 5'-CAGAACCAAGCAGACTACGC-3' and reverse, 5'-TTCCATCTCCCACCACAGTC-3'); and glyceraldehyde 3-phosphate dehydrogenase (GAPDH) (forward, 5'-TCCAGAACATCATCCCTGCC-3' and reverse, 5'-GCCTGCTTCACCACCTTCTT-3'). The target gene expression level was normalized to that of the housekeeping gene (GAPDH), and relative gene expression was calculated using the comparative $\mathrm{Ct}$ method. One sample group $(N)$ was collected from six devices $(n=6)$. Four samples $(N=4)$ were collected from a total of 24 devices.

\section{Measurement of duct permeability}

A $10-\mu \mathrm{M} 40-\mathrm{kDa}$ fluorescein isothiocyanate (FITC)-dextran solution was added to the (central) breast duct channel followed by incubation for $3 \mathrm{~h}$ at $37^{\circ} \mathrm{C}$ and $5 \% \mathrm{CO}_{2}$. Permeability was calculated according to Fick's first law:

$$
\begin{aligned}
& J=-D \frac{\partial C}{\partial x}, \\
& \text { Flux }=-P \Delta C,
\end{aligned}
$$

where $J$ is the flux, $D$ is the diffusion coefficient, $C$ is the concentration, $x$ is the position, and $P$ is the permeability. FITC-dextran diffusion was visualized from fluorescence images, and the distribution was analyzed using ImageJ software (Fig. S7).

\section{Statistical analysis}

The results are presented as the mean \pm standard error. Mean differences were evaluated with Student's $t$ test or by one-way analysis of variance. $P<0.05,0.01$, and 0.001 were considered statistically significant.

\section{Results and discussion}

\section{Physical properties of COL1 hydrogel coated with COL1}

A mammary duct is a complicated 3D structure (Fig. 1a) with 3D epithelium on the ECM (interstitial collagen and basement membrane). The basic structure of the mammary duct was mimicked by the microfluidic device, with cultured epithelial cells on the spatially modified COL1 hydrogel (Fig. 1a-c). After crosslinking, the COL1 hydrogel forms a self-assembled matrix of COL1 nanofibers that are hundreds of nanometers in diameter. Water/ medium fills the interfiber space, creating pores that are tens of microns in size ${ }^{36}$. The COL1 hydrogel has been widely used as a basic ECM for 3D cell culture due to its abundance in the human body and the fact that its mechanical properties can be easily regulated by altering the density and $\mathrm{pH}$ prior to the crosslinking step $^{37}$. 
(a)

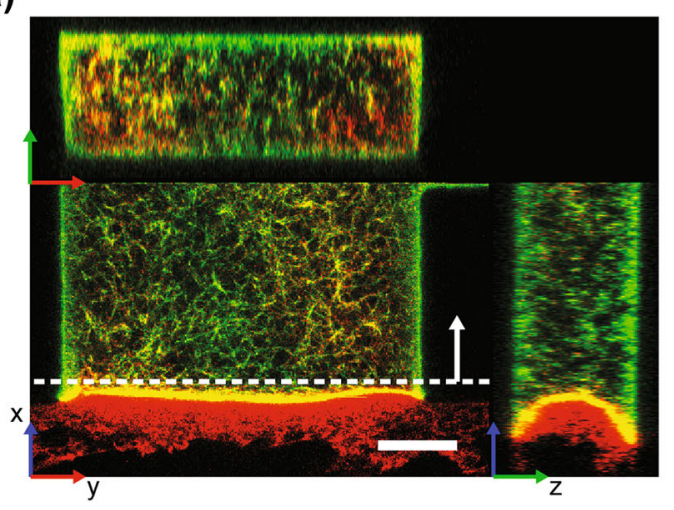

(c)
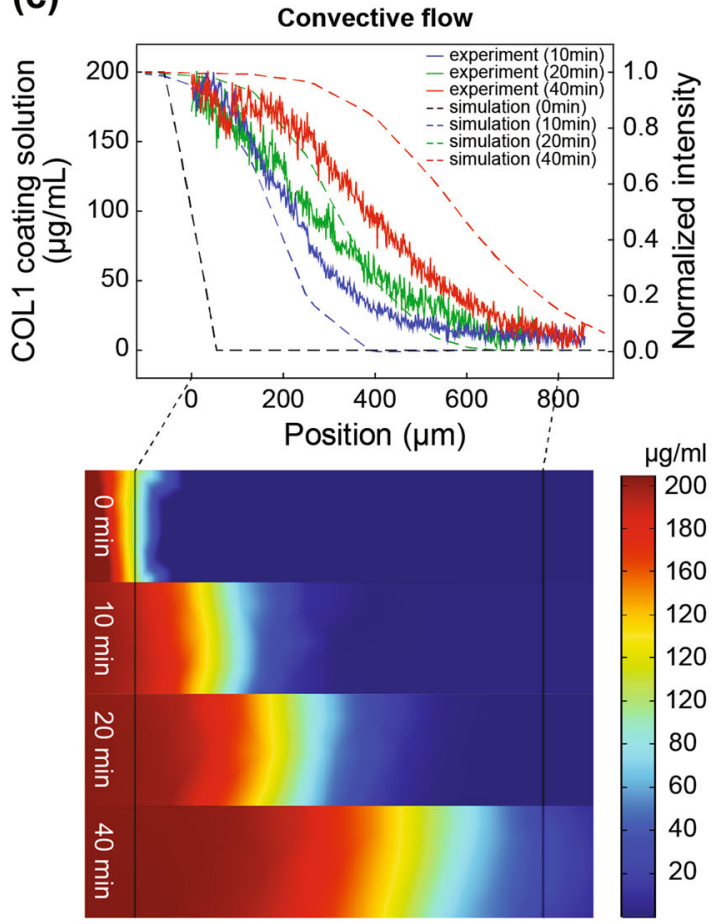

(b)

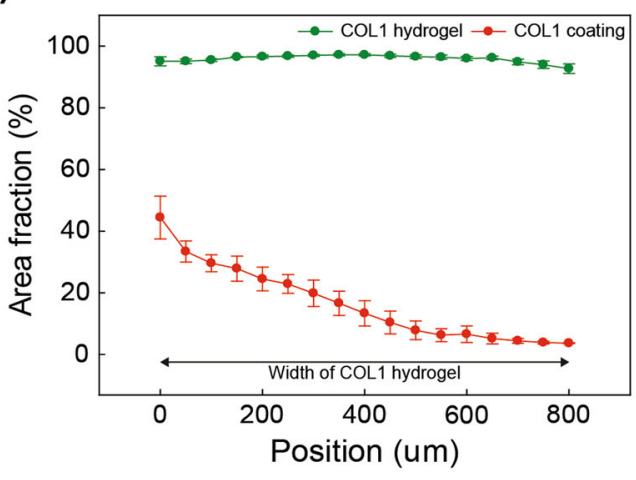

(d)

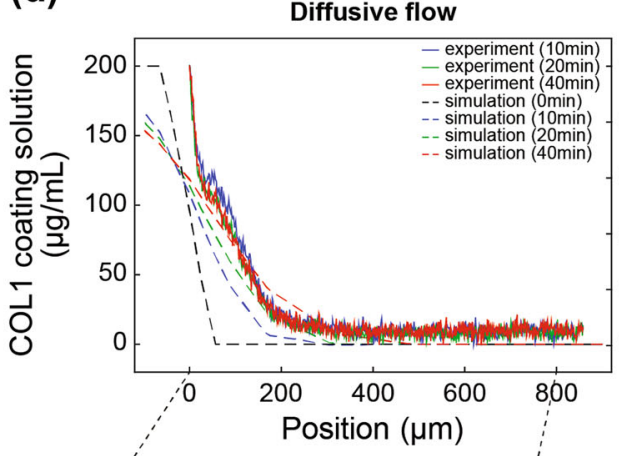

Fig. 2 Comparison of convective flow and diffusive flow coating in the microfluidic device. a Orthogonal view of labeled and spatially modified COL1 components of COL1 nanofibers from the pre-existing COL1 hydrogel (green) and from COL1 coating solution (red). The white dashed line indicates the starting point of spatially modified COL1 hydrogel. Scale bar, $50 \mu \mathrm{m}$. $\mathbf{b}$ Area fractional distribution relative to the background fluorescence in confocal images upon incubation with convective coating of COL 1 solution $(200 \mu \mathrm{g} / \mathrm{ml})$ for $40 \mathrm{~min}(n=3)$. Error bars indicate standard error. $\mathbf{c}$, d Computational simulation and experimental results of COL 1 concentration at various incubation times ( $0,10,20$, and 40 min) after application of convective (c) and diffusive (d) coating. Simulation and experimental values are indicated on the left (collagen concentration) and right (normalized intensity) axes, respectively. The position from the white dashed line in a was determined in the direction of the white arrow

Convective or diffusive flow of the $200 \mu \mathrm{g} / \mathrm{ml} \mathrm{COL1}$ coating solution was generated into the COL1 hydrogel (Fig. 1d and Fig. S1(b)). The additional COL1 coating solution cannot be visualized by immunofluorescence labeling, which was applied to differentiate MAT or laminin on COL1 nanofibers (Fig. S5(c)). We therefore labeled the original COL1 nanofibers with Alexa Fluor
488 NHS ester (green) after precuring of COL1 (COL1 hydrogel), while the COL1 coating solution was prepared by conjugation of COL1 with TRITC (red) following an established protocol $^{35}$ (Fig. 2a). After gelation, the distribution of the COL1 coating solution was visible in cross-sectional confocal microscopy images $(y-z$ plane), and the relative concentrations were calculated as the area 
of green or red color relative to the background (Fig. $2 \mathrm{~b}$ and Fig. S1(c)). The COL1 coating solution was spatially modified at the peripheral sites and sparsely distributed on the entire hydrogel, even after convective coating. The experimental distribution of the COL1 coating solution showed good correlation with the computational simulation (Fig. 2c, d). The COL1 coating solution primarily consists of the COL1 heterotrimer with large molecular weight (approximately $345 \mathrm{kDa}$ ) and a low diffusion coefficient in water $\left(7.3 \times 10^{-12} \mathrm{~m} / \mathrm{s}\right)$ (Fig. $2 \mathrm{~d}$ and Table S1). The COL1 coating solution could not be easily delivered through the pores among the COL1 nanofibers by diffusion only. Convective flow of the COL1 coating solution increased the transport of COL1 heterotrimers, allowing them to be actively delivered into the COL1 hydrogel. However, even when delivered by convective flow, the COL1 coating solution was also localized at peripheral sites. A difference between the simulation and experimental results (Fig. 2c in $40 \mathrm{~min}$ ) was also noted, mainly because of partial curing of COL1 heterotrimers on existing COL1 nanofibers during the coating process, which could not be considered in the simulation (Fig. 2c and Fig. S1(b-ii)). Therefore, COL1-coated nanofibers formed by even convective flow were observed at the periphery of the COL1 hydrogel beneath the mammary duct channel (Fig. S2). The thickness of the COL1-coated area increased with incubation time.

In the TEM images, peripheral sites of the COL1 hydrogel incorporated in the microfluidic device were located in the region near the mammary duct channel, and the central sites were located in the region at the center of the COL1 hydrogel (Fig. 3a). COL1-coated nanofibers showed an increased diameter from 0.09 to $0.21 \mu \mathrm{m}$ and a decreased interfiber space from 1.32 to $0.33 \mu \mathrm{m}$ at the peripheral sites. Interestingly, neither the diameter nor the interfiber space was altered at the central sites, and both had values similar to those of the noncoated nanofibers. The COL1 coating increased the diameter and number of COL1 nanofibers at the peripheral sites of the hydrogel, which meant that the COL1 heterotrimers coated the existing COL1 nanofibers and formed new nanofibers at the same time (Fig. 3b-d). When HMECS were cultured on the COL1-coated COL1 hydrogel, new nanofibers were added beneath the HMECs with a slightly decreased interfiber distance. This result suggests that HMECs remodel the neighboring ECM by forming new ECM nanofibers (Fig. 3b-d).

\section{Mammary ducts on various ECM hydrogels}

The ECM properties at the peripheral sites were analyzed under various ECM and ECM coating conditions (Fig. 3e-g and Fig. S4). Under COL1 hydrogel conditions (noncoated as control, high-density, and stiff COL1), the nanofiber diameter slightly decreased for high-stiffness COL1, which enhanced the stiffness of the COL1 hydrogel (a higher $\mathrm{pH}$, i.e., $\mathrm{pH} 11$, of the COL1 solution before gelation induced the formation of COL1 nanofibers with a smaller diameter and increased stiffness) ${ }^{37}$. The nanofiber diameter of the high-density COL1 hydrogel was almost same as that of the noncoated COL1 hydrogel. However, the interfiber space decreased with an increasing number of nanofibers. When the COL1 hydrogel was coated with the COL1 coating solution, the number of nanofibers increased, and the interfiber space decreased with COL1 coating concentration. The COL1 coating also increased the diameter of the COL1 nanofibers, demonstrating that the COL1 coating generated new nanofibers and thickened the existing nanofibers at the same time. MAT coating also generated new nanofibers in the COL1 hydrogel but without increasing the average diameter of the COL1 nanofibers. The coating efficiency of the MAT coating solution could be considered lower than that of the COL1 coating solution.

When HMECs were cultured under various ECM and ECM coating conditions, HMECs formed an epithelial layer with tight cell-cell junctions. The following is a summary of the ECM and coating conditions: noncoated normal COL1 hydrogel (as control, $2 \mathrm{mg} / \mathrm{ml}, \mathrm{pH} 7.4)$, noncoated high-density COL1 hydrogel $(4 \mathrm{mg} / \mathrm{ml}, \mathrm{pH} 7.4)$, noncoated stiff COL1 hydrogel $(2 \mathrm{mg} / \mathrm{ml}, \mathrm{pH} 11)$ and MAT-coated, laminin (LN)-coated, and COL1-coated normal COL1 hydrogel ( $2 \mathrm{mg} / \mathrm{ml}$, pH 7.4) (Fig. 4a).

As depicted, sprouting was reduced in HMECs cultured on high-density and stiff COL1 hydrogels. However, HMEC spouting was not blocked by rBM formed by MAT or LN coating. This result was far from expected because laminin and COL4 are major components of the mammary duct BM. Interestingly, HMECs showed decreased sprouting when COL1 was coated on the COL1 nanofibers (Fig. 4b). The concentration of COL1 coating solution also influenced the suppression of HMEC sprouting (Fig. S5(a) \& (b)). In the optimized COL1 coating protocol, HMEC sprouting was fully stabilized to form a confluent and tight mammary duct. HMECs seeded on the laminin-free COL1 hydrogel secreted laminin with a thickness and distribution that were similar to those of the optimal rBM formed by MAT coating (Fig. S5(c)). The COL1 coating on COL1 nanofibers appears to assist HMECs in remodeling of COL1 by secreting BM components. Laminin and integrin expression were visualized by immunofluorescence labeling. Compared with various ECM and coating conditions, laminin was continuously expressed in the COL1-coated COL1 hydrogel with HMECs. Laminin binds integrin on epithelial cells, thus altering cell polarity and enhancing epithelial function ${ }^{38}$. We confirmed that integrin was localized at the sites of 
(a)
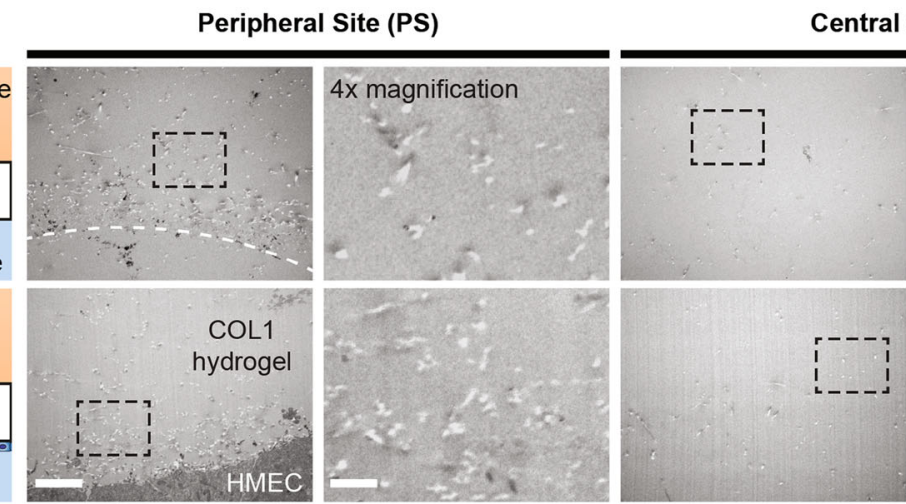

Central site (CS)
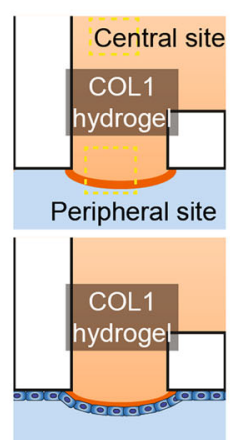

(b)

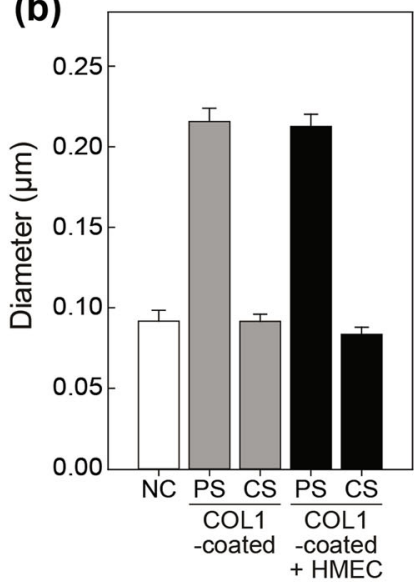

(e)

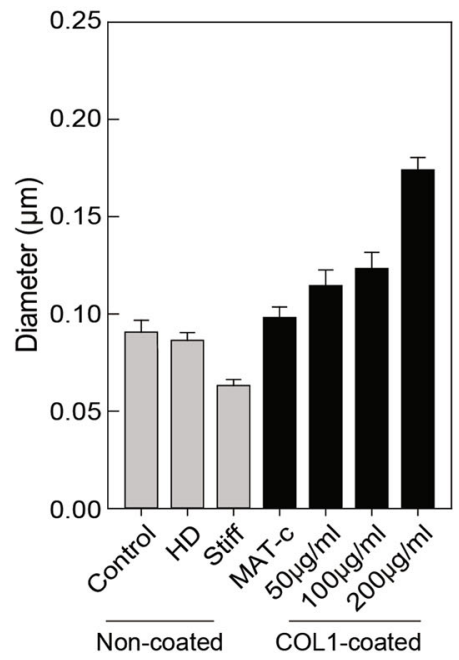

(c)

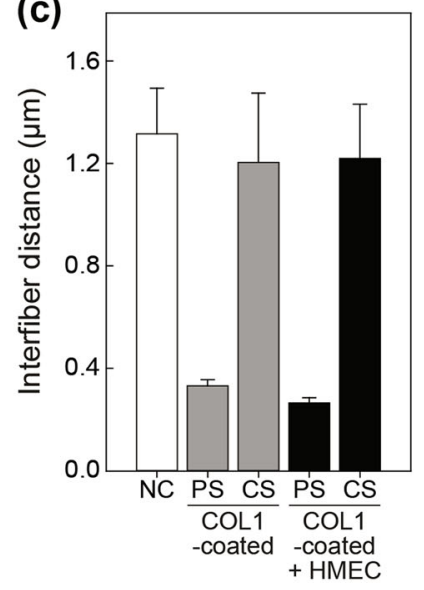

(f)

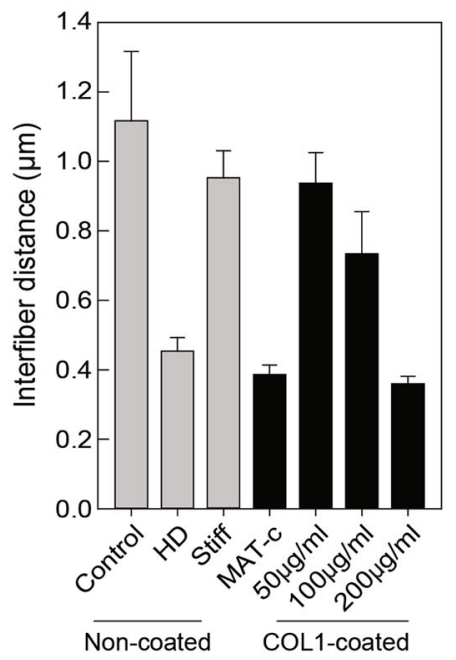

(d)

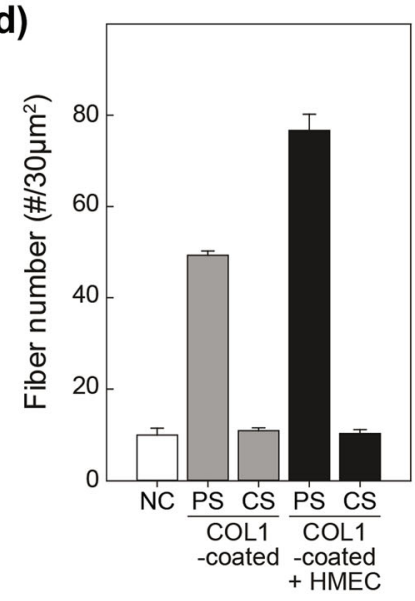

(g)

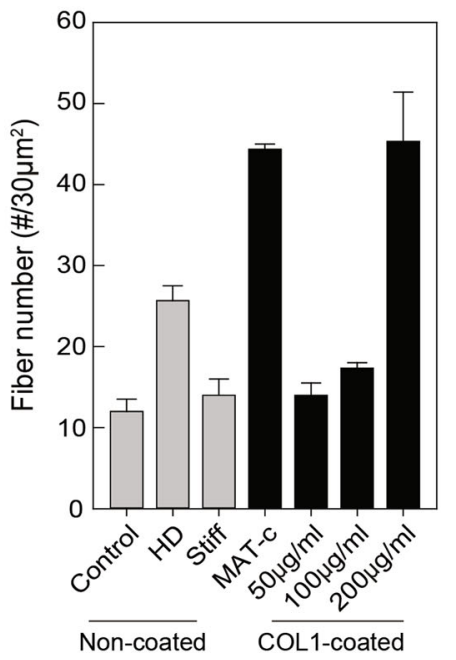

Fig. 3 Physical properties of collagen fiber under various ECM conditions. a Transmission electron micrographs of cross-sections of spatially modified COL1 hydrogel with or without HMECs at a peripheral site (PS) and central site (CS) in COL1-coated COL1 hydrogel. White dashed line indicates the interface between the COL1-coated COL1 hydrogel and mammary duct channel, and black dashed boxes indicate $\times 4$ magnification position. Scale bars, 2 and $0.5 \mu \mathrm{m}$, respectively. $\mathbf{b}$ - $\mathbf{d}$ Collagen fiber properties (fiber diameter, interfiber distance, and fiber number per $30 \mu \mathrm{m}^{2}$ area for noncoated (NC), COL1-coated, and COL1-coated with HMECs; $n=3)$. Error bars indicate standard error. Nanofibers were analyzed at peripheral and central sites. e- $\mathbf{g}$ Collagen fiber properties at peripheral site (fiber diameter, interfiber distance, and fiber number per $30 \mu^{2}$ area under various ECM and ECM coating conditions; $n=3$ ). HD indicates high-density and MAT-c indicates MAT-coated 
(a)

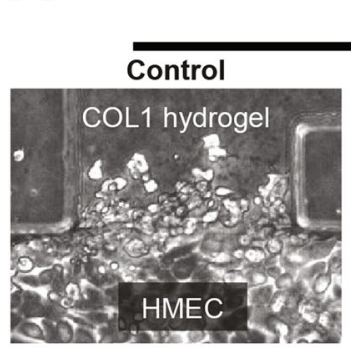

LN-coated

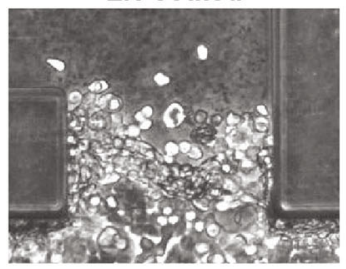

Non-coated HD

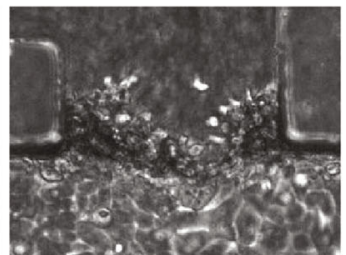
MAT-coated

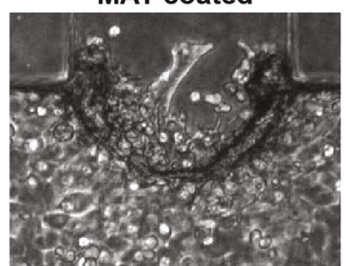

(b)

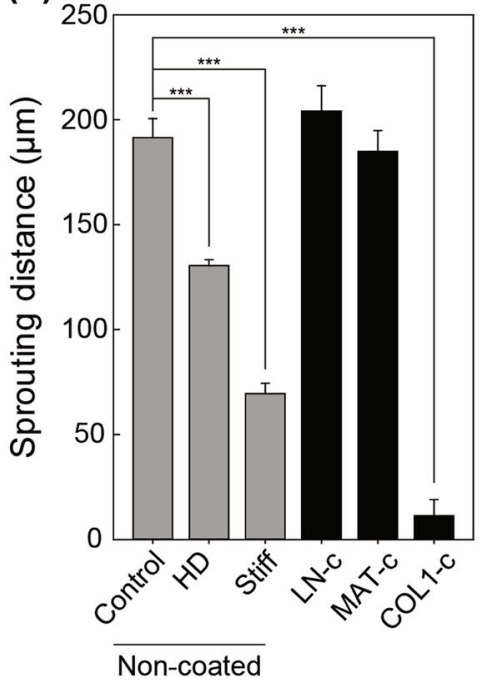

(c)
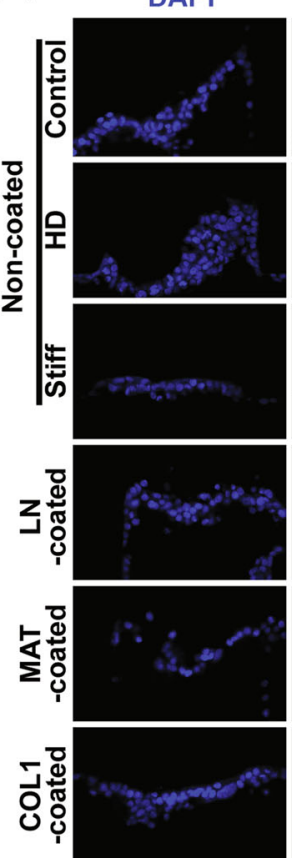

Laminin
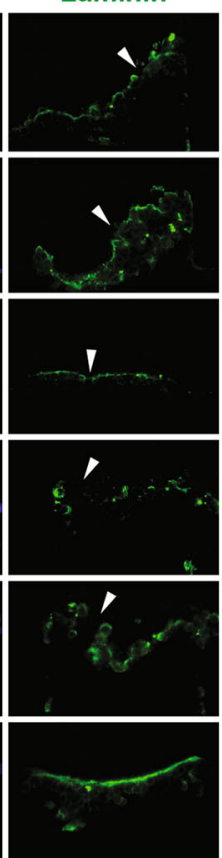

a6-integrin
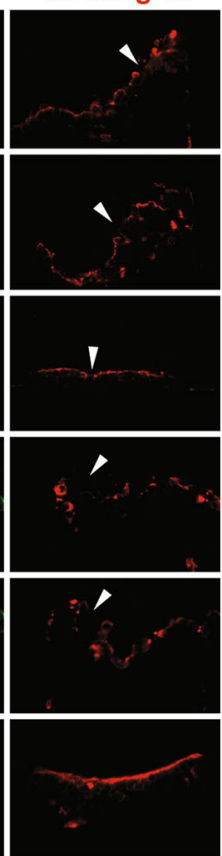

Stiff

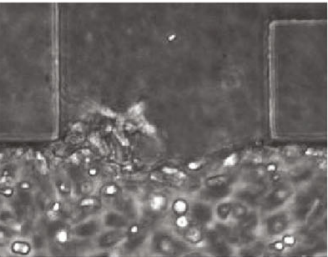

COL1-coated

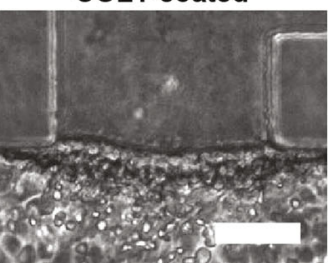

(d)
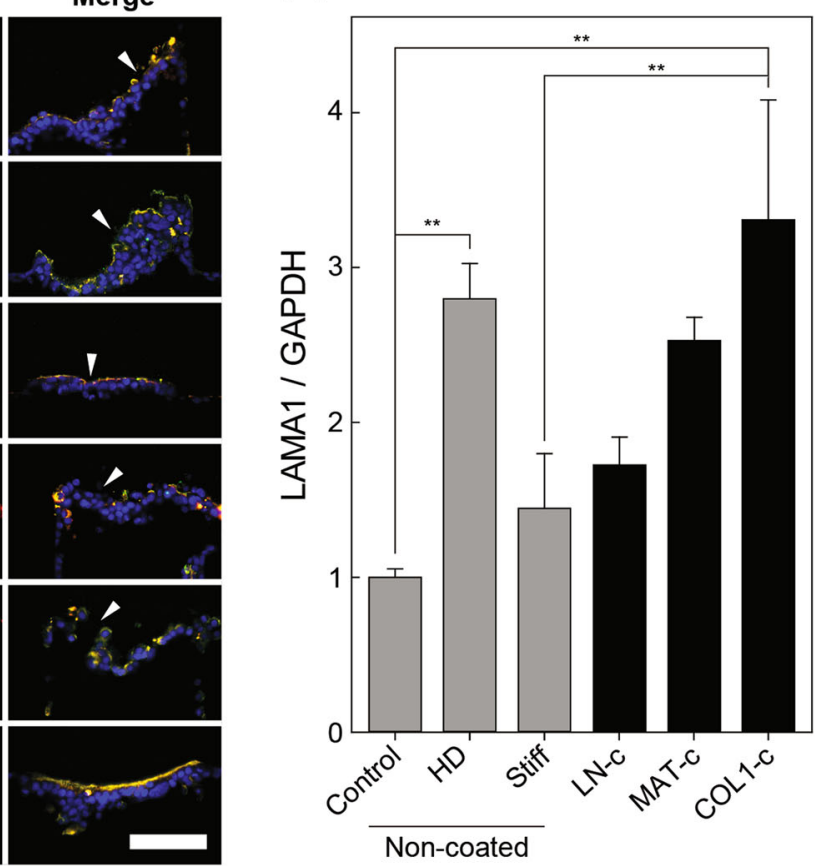

Non-coated

Fig. 4 Mammary epithelial cell morphology and basement membrane localization under various ECM hydrogel. a Phase-contrast micrographs of HMEC morphology under various COL1 hydrogel conditions with LN, MAT, and COL1 coating. Scale bar, $100 \mu \mathrm{m}$. b Sprouting distance of HMECs into COL1 hydrogel of noncoated normal (as control, $2 \mathrm{mg} / \mathrm{ml}, \mathrm{pH} 7.4)$, high-density (4 mg/ml, pH 7.4) and stiff (2 mg/ml, pH 11) COL1 hydrogel and various coating conditions on normal COL1 hydrogel $(2 \mathrm{mg} / \mathrm{ml}, \mathrm{pH} 7.4)(n=8)$. Error bars indicate standard error, and sprouting distance was normalized to the value under noncoated normal COL1 conditions ( ${ }^{* *} P<0.001, n=8$ ). c Immunofluorescence detection of laminin and a6-integrin distribution under various ECM conditions. White arrow indicates discontinuous locations of laminin and a6-integrin. Scale bar, $100 \mu \mathrm{m}$. d LAMA1 mRNA expression levels under various ECM conditions. Error bars indicate standard error. Data were evaluated by one-way analysis of variance followed by Tukey's post hoc multiple comparisons test $\left.{ }^{* *} P<0.01, N=4(n=24)\right)$. HD indicates high-density, and LN-c, MAT-c, and CO1c indicate LN-, MAT-, and COL1-coated, respectively 
(a)

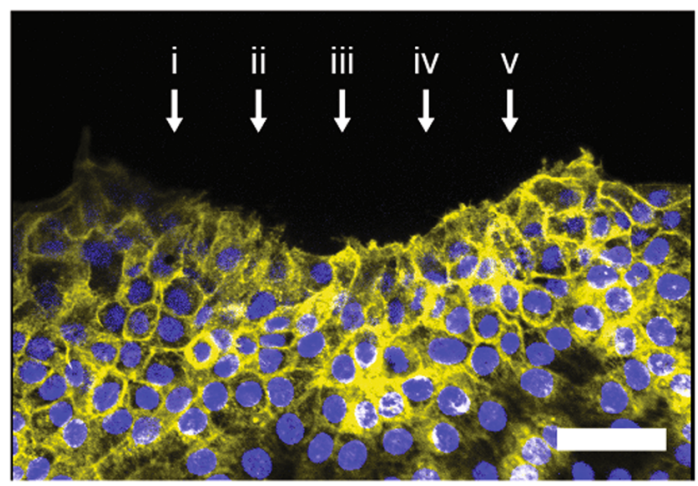

(b)

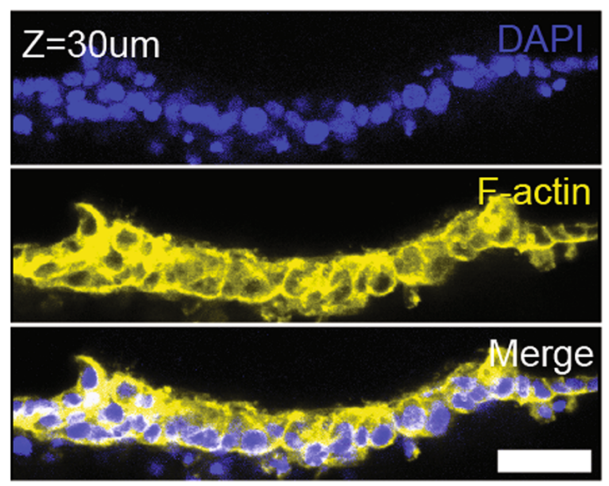

\section{(c)}
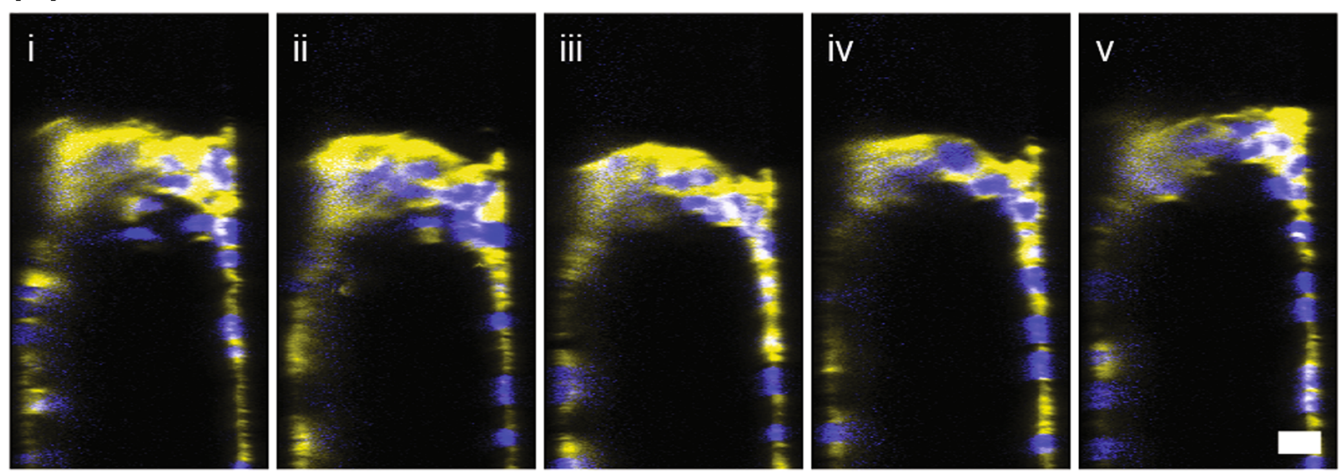

Fig. 5 Lumen structure of 3D mammary epithelium. a Confocal micrographs of HMECs on COL1-coated (200 $\mu \mathrm{g} / \mathrm{ml})$ COL1 hydrogel (under optimized coating condition). HMEC were tightly clustered in the microfluidic device. Scale bar, $50 \mu \mathrm{m}$. b Horizontal sectional views (at $z=30 \mu \mathrm{m}$ ) of stable mammary duct formed on the spatially modified COL1 hydrogel under optimized coating condition. Scale bar, $50 \mu \mathrm{m}$. c Five sequential cross-sectional views ( $i \sim v$ from fig. 5a) of the lumen structure. HMECs were stained with 4',6-diamidino-2-phenylindole (DAPI, blue) and F-actin (yellow). Scale bar, 20 um

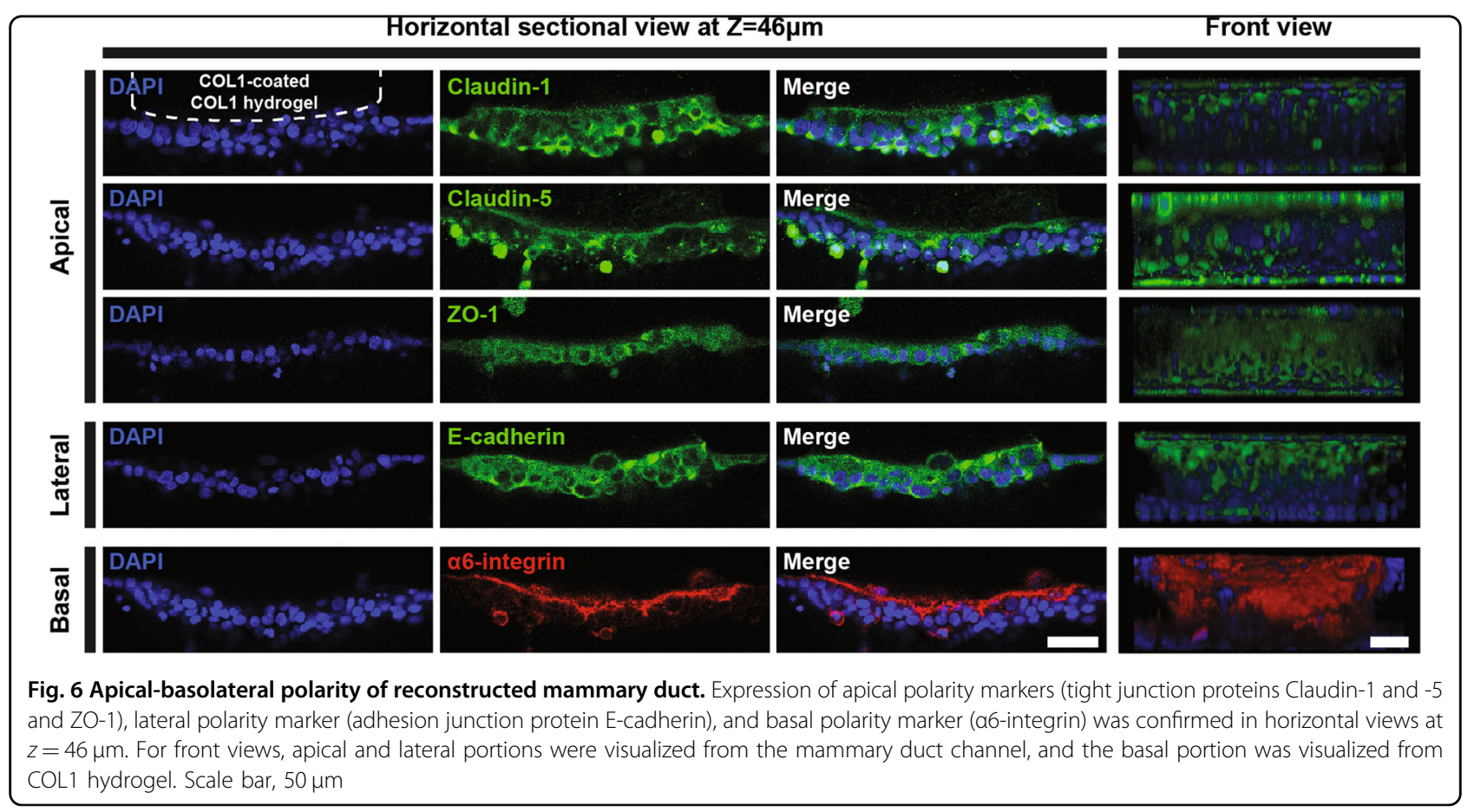


(a)

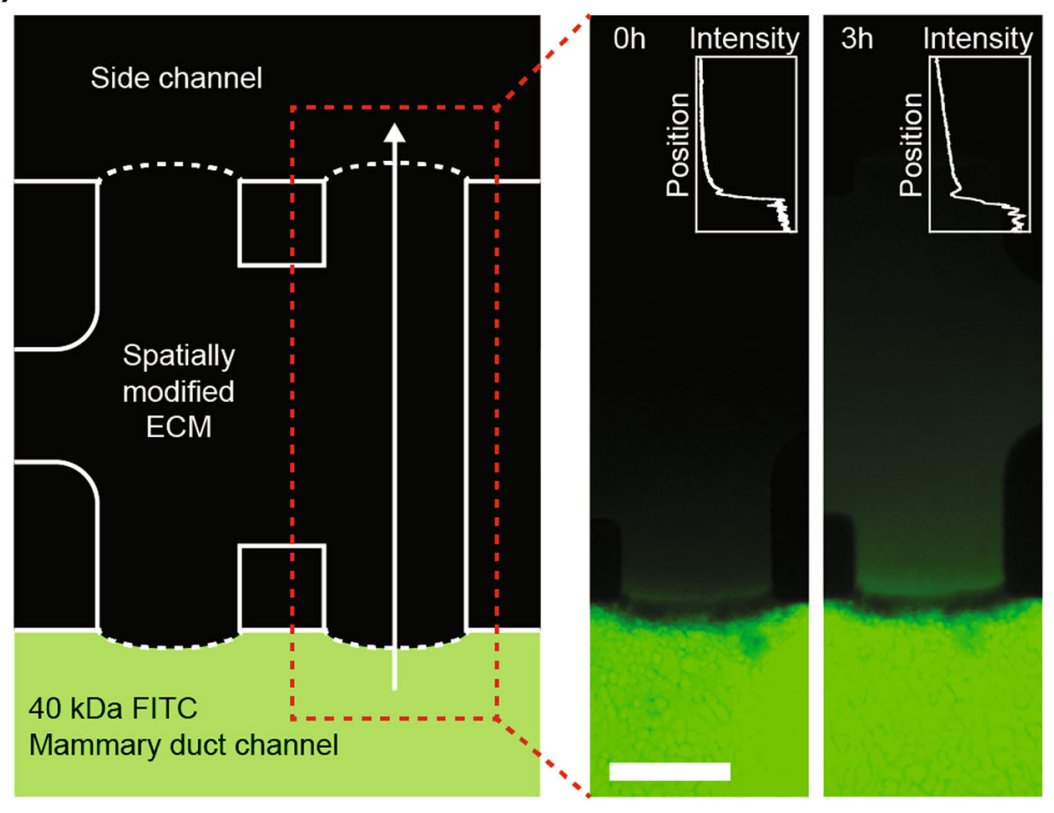

(b)
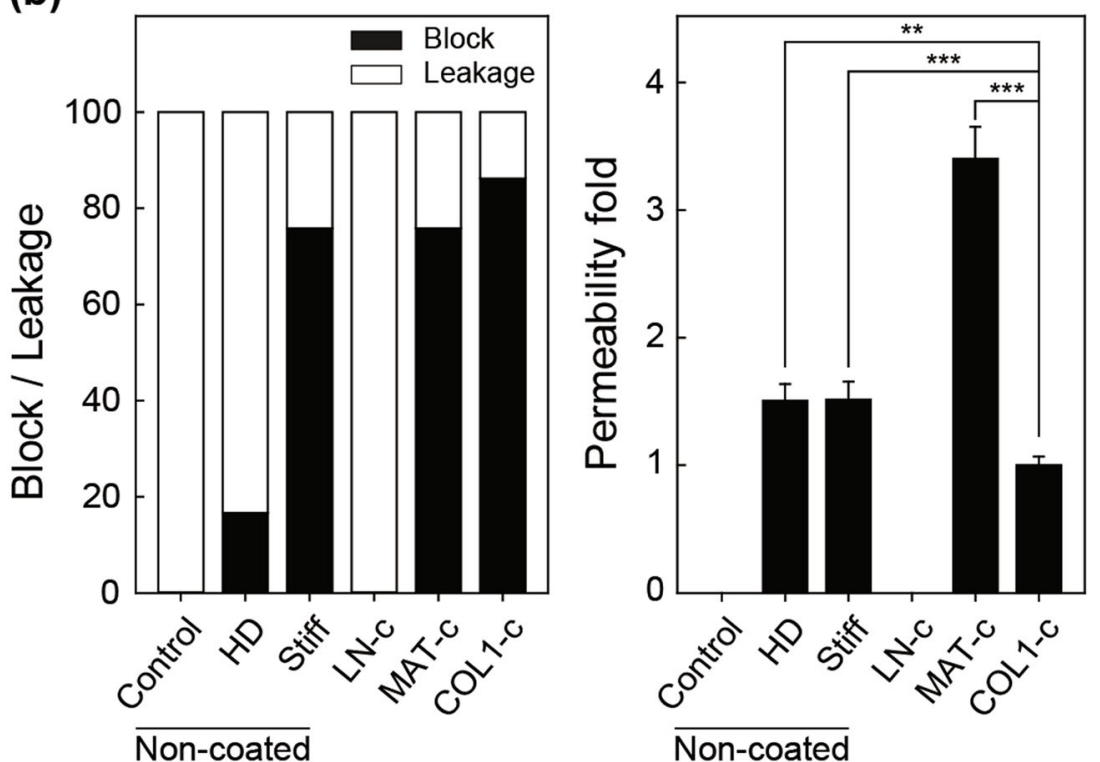

Fig. 7 Permeability of 3D mammary epithelium under various ECM. a Schematic illustration of mammary duct permeability measurement (left). FITC-dextran $(10 \mu \mathrm{M})$ in cell culture medium was introduced into the mammary duct channel (center), and the fluorescence intensity was monitored $3 \mathrm{~h}$ later (right). Inset shows FITC intensity profile from the mammary duct channel into the COL1 hydrogel (white arrow). Scale bar, $200 \mu \mathrm{m}$. b Comparison of blockage/leakage ratio (left) and relative permeability value (right) under various coating conditions. White and black bars indicate leakage and blockage ratios, respectively. Relative permeability values (means \pm standard errors) are compared with those under optimal conditions (COL1-coated $(200 \mu \mathrm{g} / \mathrm{ml})$ COL1 hydrogel) ${ }^{* *} P<0.01$ and $\left.{ }^{* *} P<0.001, n=29\right)$. HD indicates high density, and LN-c, MAT-C, and CO1-c indicate LN-, MAT-, and COL1-coated, respectively

laminin expression in the COL1-coated COL1 hydrogel with HMECs (Fig. 4c). HMECs were reported to secrete more laminin when cultured on ECM with a high density $^{15}$. We found that the spatially modified COL1 hydrogel with COL1 coating solution suppressed new sprouting and promoted HMECs to secrete laminin and remodel COL1 by formation of stable BM. This observation was confirmed by the high mRNA expression level of LAMA1 on the COL1-coated COL1 hydrogel (Fig. 4d). Since human laminin subunit alpha 1 , one of the laminin 
chain components, is encoded by LAMA1 gene, secretion of laminin from the cells was indicated by $L A M A 1$ expression.

\section{Structure and function of reconstructed mammary duct}

The 3D lumen-like epithelial structure formed by HMECs grown on spatially modified COL 1 was visualized in sequential cross-sectional images acquired on a confocal microscope (Fig. 5a-c). HMECs grown on a COL1coated COL1 hydrogel formed tight connections without leakage, while HMECs grown on noncoated, MAT- or LN-coated COL1 hydrogel were loosely connected (Fig. S6). The apical polarity of the formed epithelium was evaluated based on expression of the tight junction proteins Claudin- 1 and -5 and ZO- 1 , while lateral and basal polarity were verified by immunofluorescence analysis of E-cadherin (adhesive junction protein) and a6-integrin (ECM-epithelial cell interaction protein) expression (Fig. 6). The mammary duct was confirmed to be 3D and lumen-like, and the apical-basolateral polarity allowed directional transport of molecules passing through the epithelium.

Milk produced from the mammary gland flows to the nipple through the mammary duct. Milk leakage through the epithelium to the breast tissue can serve as a measure of the barrier function of the mammary duct. Permeability was measured using $10 \mu \mathrm{M}(40 \mathrm{kDa})$ FITC-dextran to mimic $\beta$-casein, a component of human milk. The change in fluorescence intensity was monitored immediately and $3 \mathrm{~h}$ after FITC-dextran solution filling, and the permeability was calculated according to Fick's first law ${ }^{34}$ (Fig. 7a). The blockage and leakage ratios were determined based on the rapid decrease in fluorescence intensity near the HMEC epithelium (Fig. S7). The blockage ratio indicates the probability of nonleaked mammary duct formation and is calculated with the following equation: blockage ratio $=$ blocked cases/total cases. The permeability value was calculated from cases of blockage only. The mammary duct formed on the COL1coated COL1 hydrogel had the highest blockage ratio $(86 \%)$ and the lowest permeability value $\left(3.49 \times 10^{-8} \mathrm{~m} / \mathrm{s}\right)$ (Fig. 7b), with an optimal concentration of $200 \mu \mathrm{g} / \mathrm{ml}$ COL1 coating solution (COL_200, Fig. S8). Interestingly, rBM formed by MAT coating did not enhance the permeability of the HMEC epithelium, with a value that was 3.5 times higher than that of the COL1 coating condition, despite the significant enhancement in permeability of the endothelial monolayer ${ }^{34}$. An increased concentration of MAT coating solution slightly increased the blockage ratio of the HMEC epithelium (Fig. S8), but we concluded that $\mathrm{rBM}$ did not enhance the permeability of the epithelium of the mammary duct.

As expected in the hypothesis, the physical attributes of the COL1 nanofiber were presumed to directly affect laminin secretion and mammary epithelium stability. On the optimally modified COL1 hydrogel with COL1 coating solution, HMECs chemically remodeled the neighboring ECM nanofibers by secreting BM components and forming new ECM nanofibers, creating a clear BM layer and a stable and confluent epithelium.

\section{Conclusion}

In this study, we developed a novel method for constructing a spatially modified ECM, the properties of which can be controlled in a 3D microfluidic device. Using this method, the interaction between the ECM and cells can be evaluated from the standpoint of matrix properties. In the case of mammary epithelium, regulation of the physical properties of COL1 nanofibers influenced the cellular characteristics of the epithelium to a greater extent than other coatings with BM components (MAT or LN). Mammary epithelial cells secreted more LN on COL1-coated COL1 nanofibers than on COL1 coated with BM components and formed a clearer BM layer. The cells subsequently formed a morphologically and functionally more stable mammary duct. We failed to characterize the rheological properties of the spatially modified COL1 hydrogel due to the closed nature of the microfluidic platform. In future work, hydrogelembedded fluorescent beads ${ }^{39}$ and optical magnetic twisting cytometry ${ }^{40}$ could be good candidates for investigating the distribution of the mechanical properties of the spatially modified COL1 hydrogel. We successfully constructed a physiologically relevant in vitro 3D mammary duct model that exhibited morphogenesis, apicalbasolateral polarity, barrier function, and a self-generated BM in microfluidic assay format. The model can be used to study ductal carcinoma in situ, responses to drug treatment, and interactions between the mammary duct and stromal cells (adipocytes and fibroblasts) or blood vessels.

\section{Acknowledgements \\ The authors thank Nak Won Choi from the Korea Institute of Science and Technology for technical assistance in preparing the TRITC-conjugated COL1. This work was supported by National Research Foundation of Korea grants funded by the Korean government to S.H.L. (No. 2013R1A1A2058789) and S.C. (NRF-2017R1A2B3007701 and 2014M3A7B4052193). \\ Author details \\ 'Department of IT Convergence, Korea University, 145 Anam-ro, Seoungbuk- gu, Seoul 02841, South Korea. ${ }^{2}$ Department of Radiology, Seoul National University Hospital, 101 Daehak-ro, Jongno-gu, Seoul 03080, South Korea. ${ }^{3}$ School of Mechanical Engineering, Korea University, 145 Anam-ro, Seoungbuk-gu, Seoul 02841, South Korea. ${ }^{4}$ Electron Microscopy Research Center, Korea Basic Science Institute, Chungbuk 28119, South Korea. ${ }^{5}$ Biomedical Omics Group, Korea Basic Science Institute, Chungbuk 28119, South Korea}

Conflict of interest

The authors declare that they have no conflict of interest. 


\section{Publisher's note}

Springer Nature remains neutral with regard to jurisdictional claims in published maps and institutional affiliations.

Supplementary information is available for this paper at https://doi.org/ 10.1038/s41427-018-0089-3.

Received: 1 May 2018 Revised: 12 August 2018 Accepted: 22 August 2018. Published online: 9 October 2018

\section{References}

1. Espina, V. \& Liotta, L. A. What is the malignant nature of human ductal carcinoma in situ? Nat. Rev. Cancer 11, 68-75 (2011).

2. Kalluri, R. \& Zeisberg, M. Fibroblasts in cancer. Nat. Rev. Cancer 6, 392-401 (2006).

3. Gjorevski, N. \& Nelson, C. M. Integrated morphodynamic signalling of the mammary gland. Nat. Rev. Mol. Cell Biol. 12, 581-593 (2011).

4. Griffith, L. G. \& Swartz, M. A. Capturing complex 3D tissue physiology in vitro. Nat. Rev. Mol. Cell Biol. 7, 211-224 (2006).

5. Campbell, J. J. \& Watson, C. J. Three-dimensional culture models of mammary gland. Organogenesis 5, 43-49 (2009).

6. Chaudhuri, O. et al. Extracellular matrix stiffness and composition jointly regulate the induction of malignant phenotypes in mammary epithelium. Nat. Mater. 13, 1-35 (2014)

7. Schedin, P. \& Keely, P. J. Mammary gland ECM remodeling, stiffness, and mechanosignaling in normal development and tumor progression. Cold Spring Harb. Perspect. Biol. 3, 1-22 (2011).

8. Kass, L., Erler, J. T., Dembo, M. \& Weaver, V. M. Mammary epithelial cell: influence of extracellular matrix composition and organization during development and tumorigenesis. Int. J. Biochem. Cell Biol. 39, 1987-1994 (2007).

9. Baker, B. M. et al. Cell-mediated fibre recruitment drives extracellular matrix mechanosensing in engineered fibrillar microenvironments. Nat. Mater. 14, 1262-1268 (2015).

10. Gattazzo, F., Urciuolo, A. \& Bonaldo, P. Extracellular matrix: a dynamic microenvironment for stem cell niche. Biochim. Biophys. Acta-Gen. Subj. 1840, 2506-2519 (2014).

11. Lu, P., Weaver, V. M. \& Werb, Z. The extracellular matrix: a dynamic niche in cancer progression. J. Cell Biol. 196, 395-406 (2012).

12. Karamichos, D., Brown, R. A. \& Mudera, V. Collagen stiffness regulates cellular contraction and matrix remodeling gene expression. J. Biomed. Mater. Res.Part A 83, 887-894 (2007).

13. Swamydas, M., Eddy, J. M., Burg, K. J. L. \& Dréau, D. Matrix compositions and the development of breast acini and ducts in 3D cultures. Vitr. Cell. Dev. Biol.Anim. 46, 673-684 (2010).

14. Miroshnikova, Y. A. et al. Engineering strategies to recapitulate epithelial morphogenesis within synthetic three-dimensional extracellular matrix with tunable mechanical properties. Phys. Biol. 8, 026013 (2011).

15. Lance, A. et al. Increased extracellular matrix density decreases MCF10A breast cell acinus formation in 3D culture conditions. J. Tissue Eng. Regen. Med. 10, 71-80 (2016).

16. Wolf, K. et al. Collagen-based cell migration models in vitro and in vivo. Semin. Cell Dev. Biol. 20, 931-941 (2009).

17. Pavlovich, A. L., Manivannan, S. \& Nelson, C. M. Adipose stroma induces branching morphogenesis of engineered epithelial tubules. Tissue Eng. Part A 16, 3719-3726 (2010).

18. lyengar, P. et al. Adipocyte-secreted factors synergistically promote mammary tumorigenesis through induction of anti-apoptotic transcriptional programs and proto-oncogene stabilization. Oncogene 22, 6408-6423 (2003).

19. Neve, R. M. et al. A collection of breast cancer cell lines for the study of functionally distinct cancer subtypes. Cancer Cell 10, 515-527 (2006).

20. Stull, M. A. et al. Mammary gland homeostasis employs serotonergic regulation of epithelial tight junctions. Proc. Natl Acad. Sci. USA 104, 16708-16713 (2007).

21. Grafton, M. M. G., Wang, L., Vidi, P.-A., Leary, J. \& Lelièvre, Sa Breast on-a-chip: mimicry of the channeling system of the breast for development of theranostics. Integr. Biol. (Camb.). 3, 451-459 (2011).

22. Choi, Y. et al. A microengineered pathophysiological model of early-stage breast cancer. Lab Chip 15, 3350-3357 (2015).

23. Muthuswamy, S. K., Li, D., Lelievre, S., Bissell, M. J. \& Brugge, J. S. ErbB2, but not ErbB1, reinitiates proliferation and induces luminal repopulation in epithelial acini. Nat. Cell Biol. 3, 785-792 (2001).

24. Gudjonsson, T. et al. Normal and tumor-derived myoepithelial cells differ in their ability to interact with luminal breast epithelial cells for polarity and basement membrane deposition. J. Cell Sci. 115, 39-50 (2002).

25. Bischel, L. L., Beebe, D. J. \& Sung, K. E. Microfluidic model of ductal carcinoma in situ with 3D, organotypic structure. BMC Cancer 15, 12 (2015).

26. Shin, Y. et al. Microfluidic assay for simultaneous culture of multiple cell types on surfaces or within hydrogels. Nat. Protoc. 7, 1247-1259 (2012).

27. Jeong, G. S. et al. Sprouting angiogenesis under a chemical gradient regulated by interactions with an endothelial monolayer in a microfluidic platform. Anal. Chem. 83, 8454-8459 (2011).

28. Cheng, $Y$. et al. Bioinspired multicompartmental microfibers from microfluidics. Adv. Mater. 26, 5184-5190 (2014).

29. $Y u, Y$. et al. Bioinspired helical microfibers from microfluidics. Adv. Mater. 29, 1605765 (2017).

30. Fu, F., Shang, L., Chen, Z., Yu, Y. \& Zhao, Y. Bioinspired living structural color hydrogels. Sci. Robot. 3, eaar8580 (2018).

31. Yi, B. et al. Three-dimensional in vitro gut model on a villi-shaped collagen scaffold. Biochip J 11, 219-231 (2017).

32. Wang, W., Li, L., Ding, M., Luo, G. \& Liang, Q. A microfluidic hydrogel chip with orthogonal dual gradients of matrix stiffness and oxygen for cytotoxicity test. BioChip J. 12, 93-101 (2018).

33. Yoon, J. et al. Angiogenic type I collagen extracellular matrix integrated with recombinant bacteriophages displaying vascular endothelial growth factors. Adv. Healthc. Mater. 5, 205-212 (2016).

34. Han, S. et al. Constructive remodeling of a synthetic endothelial extracellular matrix. Sci. Rep. 5, 18290 (2016).

35. Cross, V. L. et al. Dense type I collagen matrices that support cellular remodeling and microfabrication for studies of tumor angiogenesis and vasculogenesis in vitro. Biomaterials 31, 8596-8607 (2010).

36. Sapudom, J. et al. The phenotype of cancer cell invasion controlled by fibril diameter and pore size of 3D collagen networks. Biomaterials 52, 367-375 (2015).

37. Yamamura, N., Sudo, R., Ikeda, M. \& Tanishita, K. Effects of the mechanical properties of collagen gel on the in vitro formation of microvessel networks by endothelial cells. Tissue Eng. 13, 1443-1453 (2007).

38. Lee, J. L. \& Streuli, C. H. Integrins and epithelial cell polarity. J. Cell Sci. 127, 3217-3225 (2014).

39. Jang, $\mathrm{H}$. et al. Homogenizing cellular tension by hepatocyte growth factor in expanding epithelial monolayer. Sci. Rep. 8, 1-10 (2017).

40. Li, H., Xu, B., Zhou, E. H., Sunyer, R. \& Zhang, Y. Multiscale measurements of the mechanical properties of collagen matrix. ACS Biomater. Sci. Eng. acsbiomaterials. 6b00634 https://doi.org/10.1021/acsbiomaterials.6b00634 (2017). 